\title{
INTERFACES DE USUARIO EN TECNOLOGÍAS XR (EXTENDED REALITY) PARA EL APRENDIZAJE Y ENTRENAMIENTO
}

\author{
USER INTERFACES IN \\ XR (EXTENDED REALITY) \\ TECHNOLOGIES FOR LEARNING \\ AND TRAINING
}

Paula Cesarino

Grupo GEPS,

Escuela de Diseño

Industrial, Universidad

Industrial de

Santander, Colombia.

cesarinopaula95@

gmail.com

\section{Paula Galvis}

Grupo Interfaz,

Escuela de Diseño

Industrial, Universidad

Industrial de Santander,

Colombia. paula.

galvis.v@gmail.com

\section{Sara Gutierrez}

Grupo GEPS , Escuela

de Diseño Industrial,

Universidad Industrial de Santander, Colombia. saragutier94@gmail.com

\section{Alejandra Mantilla}

Grupo Interfaz, Escuela

de Diseño Industrial,

Universidad Industrial de Santander, Colombia. alejamp1296@gmail.com

\section{Laura Moreno}

Grupo Interfaz, Escuela de Diseño Industrial, Universidad Industrial de Santander, Colombia. laura_moreno_97@ hotmail.com

\section{Estefanía Reyes}

Grupo Interfaz,

Escuela de Diseño

Industrial, Universidad

Industrial de

Santander, Colombia.

estefaniareyesgarces@

gmail.com

\section{Luis Eduardo Bautista}

Rojas

Grupo Interfaz,

Escuela de Diseño

Industrial, Universidad

Industrial de Santander,

Colombia.

luis.bautista@correo.uis. edu.co 
Palabras clave

Intrefaz Gráfica de

usuario, realidad Virtual,

realidad aumentada,

atención, carga

cognitiva.

\section{Resumen}

La realidad extendida es un

conjunto de tecnologías cuyo

uso se está incrementando

rápidamente, especialmente

con el auge de las tecnologías

4.0, para el aprendizaje

y entrenamiento de

procedimientos complejos.

El objetivo de este trabajo es

identificar las características y

atributos de una interfaz gráfica

de usuario para dichos entornos

virtuales, y su influencia en el

desempeño, la atención y la

carga cognitiva del usuario. Para

lograr dicho objetivo, se siguió

una metodología de revisión

sistemática de la literatura.

Los resultados mostraron la

influencia de atributos como el

color, la forma, la separación

visual y la contigüidad espacial, espacialmente para

la señalización visual. Se

discutieron los resultados y su

posible impacto en el diseño de

interfaces gráficas para entornos

de realidad extendida.

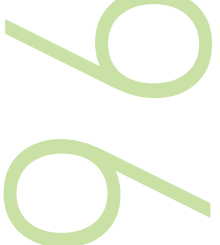

\section{Abstract}

Extended reality is a set of technologies whose use is increasing rapidly, especially with the rise of 4.0 technologies, for learning and training complex procedures. In this work, the characteristics and attributes of a graphical user interface for these virtual environments were reviewed in order to identify their influence on the user's performance, attention, and cognitive load. The results showed the influence of attributes such as color, shape, visual separation and spatial contiguity, especially for visual signaling. The results and their possible impact on the design of graphical interfaces for extended reality environments were discussed. 
Garcia-SanJuan Jaen y Nacher, (2016) afirman que aún se desconocen los efectos en el aprendiz, de la separación y presentación múltiple de fuentes de información. Finalmente, Rashid, Nacenta y Quigley (2012)

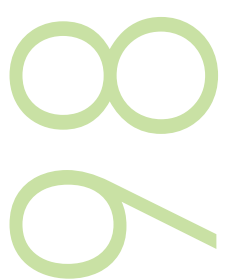
también muestran la necesidad de ampliar el estudio del efecto de atención dividida y distribución de la información, para evitar los cambios de atención visual del aprendiz

Con el fin de conocer las propiedades y atributos de los elementos de la interfaz gráfica de usuario (IGU) aplicados en la gestión de la atención visual y el manejo de la carga cognitiva en entornos extendidos, se hizo una revisión sistemática de literatura en la que se identificaron las principales características de una IGU, los atributos de los elementos más usados, los principales usos y

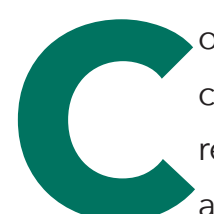

la cuarta revolución industrial, tecnologías interactivas como la realidad aumentada (RA), la realidad virtual y la realidad mixta, se vienen abriendo paso en áreas como el aprendizaje y el entrenamiento de habilidades, dada su capacidad para crear entornos sintéticos y fusionar objetos reales con virtuales en entornos de práctica simulados (Akçayır y Akçayır, 2016). El rápido crecimiento de estas tecnologías ha dado lugar a nuevos retos de diseño. Uno de ellos está relacionado con el manejo de la atención en entornos con ambientes de $360^{\circ}$ y la carga cognitiva generada por la información virtual observada, especialmente en aplicaciones de aprendizaje y entrenamiento. Teniendo en cuenta que la atención es un recurso mental limitado que influye directamente en el rendimiento y el esfuerzo, la exigencia o la carga que supone el dar una respuesta en determinada situación (Dalmau, 2008), es importante identificar las características y técnicas eficientes que ayuden a su correcta gestión. A pesar del avance en el uso de esta tecnología, pocos estudios han probado la aplicación de la RA en la educación desde la perspectiva del aprendizaje multimedia y la carga cognitiva (Lai, Chen y Lee, 2019). Estas se han centrado, particularmente, en cómo evitar los cambios de atención visual innecesarios que afectan el desempeño del aprendiz. Como lo evidencian Akçayır y Akçayır (2016), existen pocos estudios sobre los aspectos de carga cognitiva asociados a la interfaz de usuario en entornos extendidos y los existentes muestran resultados contradictorios. Por ejemplo, Ens menciona la necesidad de conocer los efectos del uso de múltiples espacios de información en un entorno de realidad aumentada, asociados al desempeño del aprendiz (Ens, Byagowi, Han, Hincapié-Ramos y Irani, 2016). De la misma forma, las herramientas de evaluación objetiva más aplicadas. Por último, se analizaron y discutieron los resultados. Asimismo, es importante aclarar que la presente investigación se desarrolla en el marco del trabajo con el semillero de investigación en Tecnologías Interactivas del Grupo de Investigación interfaz, cuyo objetivo es generar procesos formativos en investigación científica con estudiantes de pregrado en diseño industrial.

\section{Marco teórico}

La realidad extendida (o extended reality en inglés) es un conjunto de tecnologías interactivas que combinan entornos reales y virtuales junto a las interacciones humano-máquina generados por computadoras y dispositivos vestibles. Este está compuesto principalmente por la realidad virtual, realidad aumentada y realidad mixta (Fast-Berglund, Gong y Li, 2018). La realidad aumentada (RA) es una tecnología que a partir del uso de dispositivos especializados logra integrar elementos del mundo real con elementos digitales, ambos ejecutados en tiempo real (Eras y Benitez, 2007). Este mecanismo permite perfeccionar el ambiente real en lugar de reemplazarlo, razón por la cual se ha introducido en nuevos campos de investigación. Para el caso de nuestro estudio, enfocado al direccionamiento de la atención de aprendices en plataformas de realidad extendida.

\section{Realidad extendida}

La realidad virtual y otras tecnologías 3D nos están llevando hacia una realidad extendida y expandida. La realidad extendida (AR) es un término 
integral, lo que se refiere a la fusión del mundo real con el mundo virtual.

La interpretación incluye múltiples variantes (realidad mixta, realidad aumentada, virtualidad mejorada). En esta extensión de la realidad, la participación del usuario es determinante, ya que, en términos técnicos, la interacción entre estos determina el nivel de virtualidad.

De acuerdo a (Obradó Santaoliva, 2019) las características principales de las tecnologías extendidas se pueden dividir en las siguientes tres cualidades:

- Interacción: Basado en un constante feedback entre usuario - máquina/aplicación.

- Inmersión: Puede ser un proceso de simulación intelectual Obradó Santaoliva, E. (2019). Tecnologías de realidad extendida aplicadas a la creación y a la docencia artística universitaria [Universidad de Sevilla]. In Universidad de Sevilla, Sevilla (Vol. 53, Issue 9). Tesis Doctoral Inédita que implica un proceso de cambio de un estado mental a otro

- Tridimensionalidad: La disposición de objetos y elementos en las mismas coordenadas de la realidad que permite nuevas maneras de conectar y colaborar en un mismo espacio.

\section{Direccionamiento de la atención}

La orientación de la atención visual está conectada con el direccionamiento de los órganos sensoriales hacia estímulos significativos que tienen un impacto en la mejora del procesamiento de la información (Lozano, Grau, Hernández, Benaloy y Ávila, 1995). Para esto, los ojos realizan movimientos rápidos y pequeños con el objetivo de dirigirse a las señales de interés (Duchowski, 2007).

Una de las principales características de la atención visual es su constante cambio, la fluctuación en la atención depende de la disposición espacial y la complejidad de los estímulos mostrados (Barriopedro Moro, 1994; García Ogueta, 1991; Lozano et al, 1995). Debido a este rasgo dominante, los usuarios presentan cambios atencionales durante el desarrollo de las tareas realizadas en el entrenamiento procedimental desarrolladas en entornos de XR.

Con base en lo anterior, se han implementado técnicas como la codificación visual que, según varios estudios, proporciona mayor rapidez en el procesamiento de la información y gestión de la atención (Posner, 2012). Esta técnica consiste en asignar a un elemento un código visual propio (color, forma, entre otros) para indicar la categoría a la cual pertenece. Este código, identifica al elemento y permite relacionarlo por similitud con otros elementos que comparten las mismas características.

\section{Carga cognitiva}

Relacionando la complejidad de los estímulos, a los factores humanos del usuario, se menciona constantemente la teoría de la carga cognitiva, ya que esta tesis trabaja sobre el supuesto de que nuestra estructura cognitiva procesa una cantidad limitada de conocimiento (van Merriënboer y Sweller, 2005). De manera general, en la literatura, se establecen tres tipos de carga cognitiva (CC) nombrados con diversos términos. Para el presente estudio, estos últimos serán denominados de la siguiente manera: CC intrínseca, que hace referencia a la complejidad propia de la actividad (Paas, Tuovinen, Tabbers y Van Gerven, 2003); cc germánica, es aquella que conduce el aprendizaje a la memoria de largo plazo; finalmente, la cc extrínseca. Esta es causada por factores externos a la actividad y está ligada directamente a inconvenientes durante el proceso de aprendizaje y relacionada con los diseños instructivos deficientes (Sweller, van Merrienboer y Paas, 1998).

Sea como fuere, en los últimos años, varios estudios han demostrado que la sobrecarga cognitiva puede ser un aspecto importante de la usabilidad (Adams, 2007) y es probable que los entornos aumentados sean particularmente sensibles a los efectos de esta. En 2013, Harrison, Flood y Duce (2013) en su estudio sobre nuevos modelos de usabilidad plantean que este aspecto en particular (sobrecarga cognitiva) a menudo se pasa por alto en el diseño y desarrollo de productos o artefactos.

\section{Materiales y Métodos}

\section{Formación preliminar}

Con el objetivo de formar a los estudiantes en fundamentos básicos de investigación científica, se llevaron a cabo una serie de jornadas formativas y talleres, como se describe a continuación:

- Jornada 1. La investigación científica, artículos científicos y ponencias. 
- Jornada 2. Revisiones de literatura científica: bases de datos y ecuaciones de búsqueda.

- Jornada 3. Revisión de literatura: Graficación de resultados y Extracción de datos.

- Jornada 4. Escritura de resultados: Resumen y Ponencia.

\section{Reto de revisión de literatura}

Como estrategia formativa para aplicar el conocimiento mediante la formulación de un reto investigativo, se inició con una revisión de literatura descrita a continuación. La ruta metodológica de revisión está definida en los siguientes momentos: Selección de los motores de búsqueda, identificación de las palabras clave que permitan realizar las ecuaciones de búsqueda y abordar la temática de conocimiento. Posteriormente, la selección y lectura de artículos pertinentes $y$, finalmente, se hizo una bola de nieve de artículos de segundo nivel, que podrían dar respuesta a la pregunta de investigación.

Preguntas y contexto de revisión

El diseño de la interfaz gráfica de usuario en entornos de realidad extendida aplicados al aprendizaje y entrenamiento afecta el desempeño adecuado del usuario. Por ello, con el fin de conocer cuáles características de la interfaz de usuario afectan la carga cognitiva se planteó la siguiente pregunta de investigación: ¿Cuáles características de una interfaz gráfica de usuario afectan la atención y carga cognitiva de los usuarios en entornos de realidad extendida?

Una vez establecida la pregunta general de revisión, se estructuraron preguntas auxiliares de revisión, a partir de la implementación de la estrategia PICOC (Population, Intervention Comparison, Outcomes and Context, en inglés) para definir con precisión los ámbitos de búsqueda de la revisión, identificar palabras clave y crear las cadenas de búsqueda para responder la pregunta de la revisión. Así se aplicó la estrategia. En cuanto a la población, se abordaron artículos científicos, memorias de eventos premium, capítulos de libros y tesis doctorales. Con relación la intervención, se llevaron a cabo estudios experimentales con usuarios que evalúan: Rendimiento, carga cognitiva, esfuerzo mental, rendimiento, aprendizaje. En la comparación, se decidió hacer una comparación sin intervención. En cuanto al conjunto se hicieron un conjunto de estudios que definen el impacto de las características de la interfaz de usuario sobre: Rendimiento, carga cognitiva, esfuerzo mental. Por último, en la categoría contexto y nivel de cobertura, se escogieron artículos en inglés y español publicados desde 1990.

\section{Bases de datos científicas}

Los estudios científicos se identificaron mediante búsquedas en 7 bases de datos distintas: LENS, EBSCO, SCOPUS, PROQUEST, WEB OF SCIENCE Y ACM (Digital Library). Las publicaciones abarcan toda la línea de tiempo correspondiente para la existencia de cada base. Es decir, desde sus primeros artículos, hasta los más recientes (2020).

\section{Términos de búsqueda}

Se generó una lista de términos de búsqueda relacionados con los temas de la pregunta de investigación y agrupados en seis categorías como se muestra en la Tabla 1. Esta clasificación proporciona un orden para formular las ecuaciones de búsqueda en los motores de búsqueda mencionados anteriormente.

Tabla 1Términos de búsqueda

\begin{tabular}{l|l}
\hline Tecnología & Augmented reality \\
& Virtual reality \\
& Mixed reality \\
\hline Aplicación & Education \\
& Learning \\
& Training \\
& Procedural training \\
\hline Efecto cognitivo & Cognitive load \\
& Cognitive burden \\
& Workload \\
& Mental effort \\
& Attention \\
& Visual attention \\
& Attentional shift \\
& Switching of attention \\
& Split attention \\
& Divided attention \\
& Visual search \\
& Performance \\
\hline
\end{tabular}




\begin{tabular}{l|l}
\hline Señalización & Cueing \\
& Cueing Effects \\
& Visual cue \\
& Visual cueing \\
& Color cueing \\
& Signaling \\
\hline Métodos de evaluación & Electroencephalography \\
& Eyetracking \\
& EEG \\
& Saccades \\
& Fixations \\
& Areas of interest \\
& AlO \\
\hline \multirow{3}{*}{ Características } & Color \\
& Contiguity \\
& Distance \\
& Separation \\
& Panorama \\
& Field - wide \\
& Fovea- wide \\
& Layout \\
& Perspective \\
& Egocentric \\
& Exocentric \\
\hline
\end{tabular}

Fuente: Elaboración propia.

Una vez definidas las palabras clave se procede con la

combinación de términos y se plantean diferentes ecuaciones de búsqueda con el fin de obtener resultados que respondan a las preguntas de revisión.

Criterios de inclusión y exclusión

El siguiente paso fue definir los criterios de inclusión y exclusión mediante los cuales se filtraron los estudios.

Los filtros de inclusión aplicados a la búsqueda fueron los siguientes. Se tuvieron en cuenta: (1) Los documentos en idioma español o inglés, que aplicaron estudios experimentales; (2) los estudios cuya tecnología de proyección involucra entornos de realidad aumentada y realidad virtual, realidad mixta; (3) los estudios enfocados en cambios o direccionamiento de atención y atención dividida, codificación, señalización, carga mental, desempeño y aprendizaje; (4) los estudios con población adulta entre 18 a 50 años; finalmente, los estudios con métricas relacionadas con el eye-tracking.

Con el fin de obtener una búsqueda lo más relevante posible, los criterios de exclusión de los estudios aplicados a la búsqueda fueron: En primer lugar, los estudios enfocados en neurociencia o temas relacionados con medicina y patologías psicológicas; los estudios con una muestra menor de 20 participantes; los estudios con participantes menores de edad o mayores a 50 años, o con alguna deficiencia cognitiva y visual; los artículos con estudios no experimentales, sin evaluaciones con grupos de usuarios; los estudios relacionados exclusivamente con señales auditivas o kinestésicas.

\section{Selección de estudios}

Finalmente se realizó una revisión de los artículos que arrojaron las búsquedas en las bases de datos a partir de la lectura de los títulos y resúmenes. Luego, se seleccionaron aquellos que tenían estrecha relación con las preguntas de revisión. Después, se continuó con una lectura más detallada para hacer una exclusión de artículos según su contenido de acuerdo a los criterios de inclusión y exclusión. Por último, se obtuvieron los artículos completos.

En el esquema de la Figura 1 se muestra la cantidad de artículos obtenidos a partir de los distintos filtros realizados en el proceso de revisión. Por otro lado, se realizó una búsqueda no estructurada aplicando la técnica bola de nieve. Esta permite reclutar artículos de fuentes de información diferentes pero que cumplen con los criterios planteados. En esta búsqueda se obtuvieron 12 artículos que fueron incorporados a la revisión.

Figura 1 Número de los filtros de búsqued

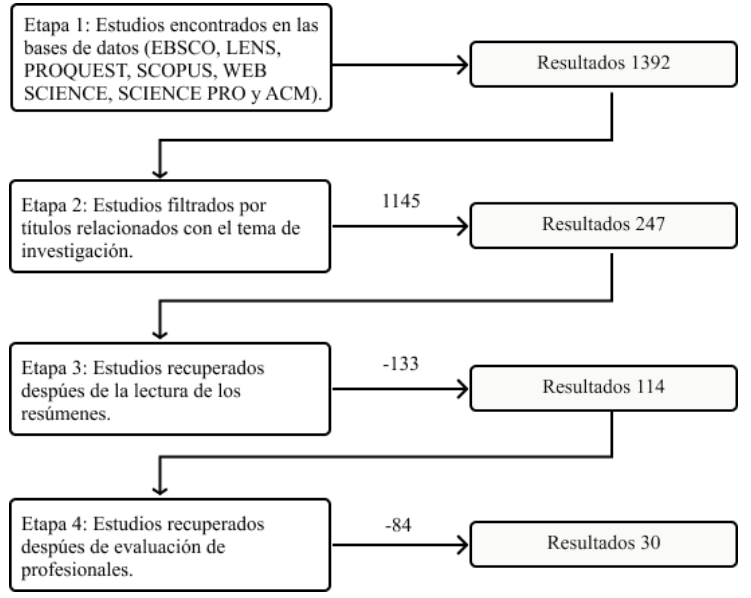




\section{Resultados}

A partir de la lectura minuciosa de los 30 artículos seleccionados, se exponen a continuación los resultados relacionados con cada una de las características y atributos evaluados relacionados con las propiedades visuales, la aplicación y uso, el manejo de la atención y reducción de carga cognitiva.

\section{Propiedades visuales}

En los seis estudios encontrados en la búsqueda con enfoque en la característica de la distancia como atributo que afecta la carga cognitiva en el proceso de aprendizaje en realidad aumentada, no se encontraron datos que relacionen medidas de distancia asociadas a la carga cognitiva. Sin embargo, se pudo identificar el principio de las instrucciones físicamente integradas a la tarea a realizar (García Ogueta, 1991; Dreyer, Oberhauser, Bandow, 2014). Ello, debido a que se evidencia que en las actividades en las que la información se encontraba en el mismo espacio físico de la tarea se obtuvieron mejores resultados de desempeño y satisfacción de los usuarios en pruebas post estudio (Schroeder y Cenkci, 2018; Johnson-Glenberg, Megowan-Romanowicz, Birchfield y SavioRamos, 2016; Mcgill, Kehoe, Freeman y Brewster, 2020). Por tanto, es posible que la carga cognitiva extrínseca se vea disminuida mediante la integración física de la instrucción (Afkari, Gómez Pérez y Bednarik, 2018).

Los artículos encontrados en la búsqueda de contigüidad espacial, no arrojaron los resultados esperados. El número de resultados obtenidos de las bases de datos en la búsqueda estructurada fueron muy reducidos y solo uno cumplió con las condiciones especificadas para esta búsqueda. A pesar de ello, se logró identificar una relación de la contigüidad espacial con el tiempo de la tarea, específicamente en estudios con pantallas 2D (Ladouce, Donaldson, Dudchenko y letswaart, 2017), en el documento se encuentra evidencia que apoya la hipótesis acerca de la importancia de la contigüidad espacial en el diseño de IGU, ya que, si la información presentada se dio en focos de atención diferentes, el tiempo en la prueba aumenta debido a la discontinuidad de la representación visual (Rashid, Nacenta y Quigley, 2012b; Rashid et al, 2012a). Es decir, si la información se presenta en focos de atención alejados espacialmente, el usuario requerirá de un mayor tiempo para la ejecución de la tarea, por el contrario, si la información se presenta en formato contiguo o distancia cercana, dicho tiempo disminuirá. Por otro lado, también se encontró que las diferencias de profundidades podrían producir una sobrecarga cognitiva en algunas tareas (Kruijff, Orlosky, Kishishita, Trepkowski y Kiyokawa, 2019).

En la revisión de literatura acerca de la perspectiva, se

seleccionaron seis estudios, los cuales corresponden con la relación de la perspectiva y la experiencia de usuario con la interfaz de realidad aumentada. En ellos se encontraron dos tipos de perspectivas, egocéntrica y exocéntrica. (Ens, Hincapié-Ramos y Irani, 2014; Keebler, Wiltshire, Smith, Fiore y Bedwell, 2014). A partir del estudio de las respectivas características de estas últimas se identificaron beneficios en ellas (Zhao, Shi, You y Zong, 2017) que dependen del entorno para el cual vayan a ser trabajadas (Anderse et al, 2016). Dicho de otro modo, si se considera que la perspectiva va de la mano con la movilidad se muestra favorabilidad en la perspectiva egocéntrica, ya que esta se moverá con el usuario (Ortega, 2013; Wang, Ong y Nee, 2016); si, por el contrario, es necesario trabajar en un entorno colaborativo, la perspectiva exocéntrica permitiría que todos sus usuarios se beneficiarán de la información allí presente (Deshpande y Kim, 2018).

En cuanto a lo que respecta al color y la forma, en 17 artículos analizados, se puede observar que la aplicación de técnicas y características de visualización sí influyen en el direccionamiento de la atención de manera positiva. Asimismo, algunos de los estudios demuestran que el uso de codificación por color logra mejores resultados en el rendimiento de los usuarios comparado con otras técnicas como lo evidencian Folker y Ritter (2005). En dichos casos, la codificación por color en el material de aprendizaje tuvo un menor tiempo de procesamiento de la información en comparación con el apoyo en texto sin ningún tipo de ayuda visual, también se observa que el uso de señales de color en controles manuales hace parte de la eficiencia para reducir errores de reversión, disminuir el tiempo de procesamiento y el número y duración de fijaciones en el desarrollo de tareas simples o complejas en entornos de RA (Chintamani, Cao, Ellis y Pandya, 2010). En cuanto a la preferencia del color en entornos de RA el color verde fue el más fácil de identificar (Lou et al, 2012). Aunque en el estudio realizado por Kruiiff, Orlosky, Kishishita, Trepkowski y Kiyokawa (2019) los resultados mostraron con respecto al color de la etiqueta, que el azul produjo la preferencia más alta y se eligió con 
mucha más frecuencia que los otros colores. Fue necesario revisar las técnicas de señalización existentes que orientan la atención con el fin de observar cómo el color o la forma de las señales participaban en estas técnicas. En la mayoría de los estudios analizados, se usó el color rojo y verde para identificar las señales (Hein, Bernhagen y Bullinger, 2019; Volmer, Baumeister, Von Itzstein, Bornkessel-Schlesewsky y Schlesewsky, 2018; Dauenhauer y Muller, 2017; Eyraud, Zibetti y Baccino, 2015; Taylor, Bilgrien, He y Siegelmann, 2015). En efecto, Van Dam, Krasner y Gabbard (2020) utilizan el color rojo para el contorno de los objetivos con el fin de dirigir la atención visual. Asimismo, ponen a prueba cuatro tipos de formas diferentes, en los que la señal límite de esquina resulta ser la más útil para atraer la atención del usuario. En el caso de la técnica de embudo omnidireccional propuesta por Biocca, Owen, Tang y Bohil (2007) los planos del embudo toman forma de marcos cuadrados de color verde. Dichos planos indican la trayectoria desde el usuario hasta el objetivo, por lo que aumentan la velocidad de búsqueda visual y disminuyen la carga de trabajo mental en un $18 \%$ comparado con otras técnicas. En el estudio de Renner y Pfeiffer (2017) utilizan las líneas para conectar objetos y relacionar elementos basados en su apariencia (forma y color), lo que permite asociar más rápidamente los elementos visibles en tareas de recolección. A diferencia de estos estudios, en el estudio de Andersen et al (2016) utilizan señales en forma de aros azules aguamarinas para indicar el lugar correcto donde se deben poner unas pegatinas, para minimizar el error de ubicación en procedimientos de telementoría. Finalmente, gracias a las contribuciones de Lu, Duh y Feiner (2012), Zhao et al (2014), Kishishita Kiyokawa, Orlosky, Mashita, Takemura y Kruijff (2014) se puede concluir que las características como el contraste, transparencia, resaltados, luminosidad, tamaño, entre otras, influyen en la aplicación de técnicas visuales para orientar la atención. Por ello, tales características se deben tener en cuenta para el diseño de interfaces de realidad aumentada.

\section{Usos en manejo de la atención y reducción de carga cognitiva} A través del análisis de nueve artículos referentes a señalización visual, se encontró que, en el desarrollo de actividades diarias, así como en el momento en que el estudiante se enfrenta a un entorno multimedia, los objetivos visuales no aparecen aislados del resto de elementos del campo visual (Jang, Shin, Kim y Kim, 2016). La capacidad de dirigir la atención a los estímulos clave, es una tarea compleja y exigente, por lo que la forma en la que aparece la señalización, debe guiar al usuario a través de la interfaz gráfica de usuario (Marek y Pollmann, 2020). Para ello, se debe tener en cuenta el concepto de señalización. Este se define originalmente por Meyer (1975) como una suerte de añadidura de un aspecto del ritmo

semántico, pero sin contenido. Este enfatiza algunos rasgos en los significados o rinde cuenta de ciertos factores estructurales. Dicho de otro modo, las señales están destinadas a guiar el direccionamiento de la atención de los usuarios dentro de las interfaces gráficas, pero no a proporcionar nueva información (Rojas-Murillo y Pennathur, 2019; Koning, Tabbers y Paas, 2007).

Asimismo, la orientación de la atención en RV, debe darse con el menor costo cognitivo y un máximo desempeño, ya que el uso de señales para el proceso de orientación de la atención, mejora el rendimiento y la velocidad de procesamiento de la información visual presentada en el entorno. Esto produce beneficios (efecto de señalización significativo) y guía la atención local y global. Así, dependiendo del tipo de señal visual que se utilice (endógena y exógena), la ayuda para la mirada por medio de señales visuales reduce los sesgos y la carga cognitiva, medida por una dilatación de la pupila más pequeña. Aspecto clave a tener en cuenta, ya que las tecnologías como la RV son utilizadas actualmente para entrenamiento procedimentales guiados por dos factores esenciales: La transformación de la información declarativa en acción y la repetición a través de la práctica (Soret, Charras, Hurter y Peysakhovich, 2019).

Por último, se detecta en la revisión bibliográfica que las técnicas de atención visual comúnmente utilizadas en entornos 3D son tres: (1) Embudo de atención, técnica que utiliza affordances perceptivas dinámicas para llamar la atención del usuario por el embudo hasta la ubicación objetivo; (2) flechas, cone ellas el usuario debe seguir la dirección de la flecha hasta que el elemento de destino se superponga con el objetivo visual; (3) finalmente, se encuentran las ondas esféricas, estas son las ondas que se propagan hacia el objetivo, como los círculos concéntricos que aparecen al lanzar una piedra al lago, solo que en sentido inverso (Renner y Pfeiffer, 2017). 


\section{Evaluación de carga cognitiva}

En forma general, se encontró que los seis estudios hallados buscan

justificar y relacionar la teoría de la carga cognitiva con el aprendizaje. Estos investigan los diferentes tipos de carga cognitiva y los métodos específicos para disminuir cada una de ellas. De los artículos encontrados en la búsqueda, sólo cinco artículos contenían información relacionada a los métodos de evaluación de la carga cognitiva.

A partir del análisis de los artículos seleccionados, se determinó que la demanda de procesamiento provocada por la tarea de aprendizaje puede exceder la capacidad de procesamiento del sistema cognitivo. Situación que se denomina sobrecarga cognitiva. Para no superar la capacidad limitada de la memoria humana, la teoría de la carga cognitiva proporciona un método para crear un entorno de aprendizaje eficaz. El objetivo de este último, es reorganizar el equilibrio entre la carga germánica y la carga interna y reducir o eliminar por completo la carga extrínseca. Como método de medición de carga mental encontrado en la búsqueda, se identificaron factores como el tiempo de respuesta y el tiempo de reacción a una tarea secundaria. Adicionalmente, se menciona el uso de electroencefalograma (EEG) que hasta el momento se ha llevado a cabo principalmente en condiciones controladas de laboratorio; por ejemplo, utilizando tareas que involucran la memoria a corto plazo para la investigación. La RA ofrece una combinación del mundo virtual y real. En efecto, evidencias muestran que el uso en escenarios de educación, aumenta el rendimiento de los estudiantes y disminuye sus niveles de carga cognitiva. Al mismo tiempo, no se puede ignorar que las opiniones de los estudiantes sobre la tecnología de RA fueron positivas (Turan, Meral y Sahin, 2018).

Por otra parte, la reducción de la carga cognitiva (CL) puede promover el aprendizaje y la adquisición de habilidades. En este sentido, se determina que el desempeño de la tarea secundaria, incluida la medición del tiempo de reacción, es un método directo y objetivo establecido para determinar la CL (Andersen, Mikkelsen, Konge, Cayé-Thomasen y Sørensen, 2015). Sin embargo, se requiere mayor investigación para explorar la experiencia completa en aprendizaje desarrollado a través de RA (Cheng y Tsai, 2013). Además de lo anterior, vale la pena resaltar que los principios de coherencia, señalización, redundancia espacial y cognitiva temporal se aplican para reducir la carga cognitiva externa en el aprendizaje de anatomía de estudiantes de medicina a través de realidad aumentada móvil (RAM) (Küçük, Kapakin y Göktaş, 2016). Asimismo, la discriminación de los estados cerebrales durante la experiencia de realidad mixta es fundamental para adaptar características de datos específicos a la

actividad cerebral contingente. Esto conduce a un mejor desempeño general del análisis discriminante lineal (LDA) con respecto a las máquinas de vectores de soporte (SVM) y sugiere la aplicabilidad de

- un enfoque en un escenario de Realidad Mixta (MR) controlado por Interfaces Cerebro-Computador (BCl) (De Massari et al, 2014). Por último, se presenta evidencia de que las oscilaciones del EEG en las bandas alfa y theta reflejan el desempeño cognitivo y de la memoria en particular. El buen rendimiento está relacionado con dos tipos de fenómenos EEG, esto es, un aumento tónico en alfa, pero una disminución en el poder theta (Klimesch, 1999).

\section{Discusión}

La búsqueda sistemática de literatura que se hizo en este estudio, gira alrededor del diseño de interfaces gráficas de usuario en entornos de realidad extendida aplicados al aprendizaje. Asimismo, se centra en analizar cómo las características de estas interfaces afectan la carga cognitiva y el direccionamiento de la atención de los usuarios que las utilizan. De acuerdo con la revisión, las señales visuales son eficaces para guiar la atención visual de los usuarios-aprendices y brindar apoyo en tareas de entrenamiento procedimental. La señalización visual carece de significado por sí misma, pero proporciona a los usuarios una guía para llegar a la comprensión de contenidos con mayor facilidad. El uso de señales visuales obliga a los usuarios a dirigir la atención a la ubicación donde aparece la información relevante en el contenido multimedia (Lucas y Vuilleumier, 2008). Los resultados de los estudios de Moon y Ryu (2020) y de Taylor, Rapp y Brunye (2007) muestran que grupos de usuarios que hicieron tareas sin la presencia de señalización visual, mostraron tiempos de fijación significativamente más largos en el panel de contenido de la instrucción en comparación con aquellos con señales visuales. Por lo tanto, las señales visuales ayudan a los usuarios a reducir el tiempo necesario para el procesamiento cognitivo necesario para comprender las tareas que deben desarrollar durante el entrenamiento procedimental. 
El uso de características para la codificación visual, contigüidad en los elementos de las interfaces gráficas de usuario y elementos de señalización visual, proporcionan una mejora en la rapidez del procesamiento de la información y la gestión de las tareas a realizar dentro de los escenarios de entrenamiento procedimental. De manera general, la integración de todos los componentes descritos conlleva a una reducción de la carga cognitiva, lo cual promueve que los usuarios aprendan mejor y adquieran habilidades de manera más efectiva. Adicionalmente, varios estudios demuestran que el uso de codificación por color logra mejores resultados en el rendimiento de los usuarios comparado con otras técnicas como las anotaciones y disminuye el tiempo de procesamiento y el número y duración de fijaciones en el desarrollo de tareas simples o complejas en entornos de RA. Por otro lado, se ha identificado que la codificación por color ha sido poco aplicada para disminuir el esfuerzo mental. No obstante, esta última es altamente utilizada para atraer la atención y mejorar el rendimiento del usuario. Asimismo, se identificó que las características como el contraste, transparencia, resaltados, luminosidad, tamaño, entre otras, influyen en la aplicación de técnicas visuales para orientar la atención. Motivo por el cual se deben tener en cuenta para el diseño de interfaces de realidad aumentada.

La información físicamente integrada favorece la carga cognitiva extrínseca y el aprendizaje en general. En contraste, se debe tener en cuenta la contigüidad en el diseño de interfaz para RA y se presume que la diferencia de profundidad entre elementos de información podría tener un impacto negativo en el desempeño y el tiempo del usuario al ejecutar la tarea. En cuanto a la perspectiva se debe tener cierta claridad de cuál es el entorno para el que se está diseñando la interfaz, ya que según esto se elegirá un tipo de perspectiva más favorable para su correcto desarrollo. Al respecto, se debe tener en cuenta que la perspectiva egocéntrica es más adecuada para el uso unipersonal, mientras que la exocéntrica resulta más colaborativa.

En cuanto a la medición de la carga cognitiva, si bien la literatura reconoce la utilidad de las tecnologías emergentes, los investigadores no se han centrado en los efectos negativos que la tecnología puede traer. Así como la interactividad de los elementos determina la carga cognitiva intrínseca, también determina la carga cognitiva externa. Dado que los elementos interactuantes que generan una carga cognitiva intrínseca son inevitables si no se cambia la tarea o los niveles de experiencia, la carga externa está bajo el control de los instructores. La teoría de la carga cognitiva

(CLT) es cada vez más importante en el diseño y evaluación de la enseñanza tradicional y técnica. Aunque es ampliamente conocido como estructura, la medición de la carga cognitiva causada por los

materiales didácticos (especialmente la enseñanza multimedia) se basa principalmente en métodos indirectos, subjetivos o ambos.

\section{Conclusiones}

En este trabajo se presentaron los resultados de una experiencia de investigación formativa aplicada a un reto por estudiantes de pregrado y posgrado. Asimismo, se socializaron y discutieron los resultados del reto planteado con respecto a un tema de actualidad. Como ejercicio de formación e introducción a la investigación científica, el trabajo presentado permitió tener una experiencia concreta con relación al reto planteado que se traduce en aprender la estructuración de una búsqueda, el manejo de bases de datos y la lectura ordenada de artículos científicos, obteniendo como resultado la escritura de un artículo. Esta experiencia creó en los participantes, fuertes cimientos para la investigación futura en la disciplina del diseño industrial buscando contribuir al mejoramiento del diseño de interfaces para realidad aumentada a través del reconocimiento de características relevantes para el proceso de diseño; características que en la actualidad no se tienen en cuenta y que pueden llegar a ser factores determinantes para el correcto desarrollo de una aplicación de realidad aumentada.

El uso de aplicaciones de realidad aumentada enfocadas al aprendizaje puede presentar problemas relacionados con la carga cognitiva, ya que se generan cambios de atención constantes, niveles bajos de retención de la información, cansancio mental, entre otros, estas características se identifican con el fin aportar herramientas que favorezcan un aprendizaje más eficiente y mejoren la experiencia del usuario en su relación con las mismas. los resultados de la búsqueda permitieron identificar la señalización como uno de los principios más aplicados para captar y direccionar la atención, este se basa en la hipótesis de que las personas comprenden mejor la información 
cuando los materiales de instrucción incluyen señales que resaltan elementos relevantes o permite una mejor organización del material. Algunos de los atributos identificados fueron el color y la forma; se estableció que, al momento de la interacción, el uso de características visuales como el color y la forma tienen especial importancia en tareas de localización y búsqueda visual.

El material de contenido multimedia y la forma en cómo se presenta la información, deben estar diseñados con base al funcionamiento de la mente humana, ya que tienen más probabilidades de direccionar correctamente la atención y generar una menor carga cognitiva externa en el usuario. Es por esto que el mayor aporte se configura en exponer la importancia de un correcto diseño y configuración de interfaces en términos generales, que tenga como objetivo mejorar la experiencia e interacción del usuario, dado que este campo exige una contextualización y comprensión del tema. Así mismo, sus resultados proporcionan el conocimiento y comprensión acerca de cómo se ha abordado la presentación y evaluación de estímulos visuales en interfaces de realidad aumentada empleando diversas técnicas, señales y características visuales que afectan principalmente la atención de los usuarios; generando una base para continuar la investigación en aras de lograr configuraciones de material multimedia que permitan direccionar los cambios de atención, disminuir los niveles de carga cognitiva, procurando mejorar el desempeño por parte del usuario en la comprensión de la información presentada y obteniendo una experiencia de interacción satisfactoria.

Este análisis generó así mismo diversos interrogantes para futuros estudios, como la influencia de la iluminación en la visibilidad del color; lo que pone en cuestión el uso del color y sus propiedades (valor, tono y saturación) en escenarios con diferentes tipos de iluminación. Respecto a la forma, se observó que en la mayoría de los estudios se emplean formas similares y básicas como el círculo, el cuadrado o el triángulo y a pesar de encontrar técnicas como el embudo de atención de Biocca (2007) que proponen un concepto diferentes, de qué manera se pueden generar nuevas formas o técnicas visuales por medio de la exploración de sus propiedades (contorno, tamaño, posición, dirección) u otros tratamientos formales que puedan hacerlas más eficientes y llamativas para el usuario. Sumado a esto la investigación también abre una puerta a preguntarnos en qué medida exacta disminuiría la carga cognitiva con la aplicación de estas características; para esto sería pertinente el uso de una medición directa que proporcione mayor información de los efectos en el participante.

\section{Referencias bibliográficas}

Adams, R. (2007). Decision and stress: cognition and e-accessibility in the information workplace. Univers. Access Inf. Soc., 5(4), 363379. DOI: 10.1007/s10209-006-0061-9.

Afkari, H., de Gómez Pérez, D. G. \& Bednarik, R. (2018). Command Selection in Gaze-based See-through Virtual Image-Guided Environments. En Proceedings of the 9th Augmented Human International Conference. (pp. 1-8). Nueva York: Association for Computing Machinery. DOI: 10.1145/3174910.3174940.

Akçayır, M \& Akçayır, G. (2016). Advantages and challenges associated with augmented reality for education: A systematic review of the literature," Educational Research Review, 20, 1-11. DOI: 10.1016/j.edurev.2016.11.002.

Andersen, S. A. W., Mikkelsen, P. T., Konge, L., Cayé-Thomasen, P. \& Sørensen, M. S. (2015). Cognitive load in distributed and massed practice in virtual reality mastoidectomy simulation. Laryngoscope, 126(2), E74-E79. DOI: 10.1002/lary.25449.

Andersen, D., Popescu, V., Cabrera, M. E., Shanghavi, A., Gómez, G., Marley, S., Mullis, B. \& Wachs, J. (2016). Medical telementoring using an augmented reality transparent display. Surgery, 159(6), 1646-1653. DOI: 10.1016/j.surg.2015.12.016.

Barriopedro Moro, M. I. (1994). El desplazamiento de la atención por el campo visual: una revisión crítica," Revista de psicología general y aplicada, 47, 373--381.

Biocca, F., Owen, C., Tang, A. \& Bohil, C. (2007). Attention issues in spatial information systems: Directing mobile users' visual attention using augmented reality. Journal of management of informational systems, 23(4), 163-184. DOI: 10.2753/MIS07421222230408

Cheng K. H. \& Tsai, C. C. (2013). Affordances of Augmented Reality in Science Learning: Suggestions for Future Research. Journal of scientific education and technology, 22(4), 449-462. DOI: 10.1007/s10956-012-9405-9. 
Chintamani, K., Cao, A., Ellis, R. D. \& Pandya, A. K. (2010).

Improved telemanipulator navigation during display-control misalignments using augmented reality cues. IEEE Trans.

Syst. Man, Cybern. Part A Systems Humans, 40(1), 29-39. DOI:

10.1109/TSMCA.2009.2030166.

Dalmau, I. (2008). Evaluación de la carga mental en tareas de control: técnicas subjetivas y medidas de exigencia. Universitat Politècnica de Catalunya, 2008.

Dauenhauer, R. \& Muller, T. (2017). An Evaluation of Information Connection in Augmented Reality for 3D Scenes with Occlusion. Adjun. Proc. 2016 IEEE International Symposium on Mixed and Augmented Reality (ISMAR-Adjunct), Merida. DOI: 10.1109/ ISMAR-Adjunct.2016.0083.

Deshpande, A. \& Kim, I. (2018). The effects of augmented reality on improving spatial problem solving for object assembly. Advance engineering informatics, 38, 760-775. DOI: 10.1016/j. aei.2018.10.004.

Dreyer, D., Oberhauser, M. \& Bandow, D. (2014). HUD Symbology Evaluation in a Virtual Reality Flight Simulation. HCl-Aero 2014 - Proceeding International Conference of Human-Computer Interaction. DOI: https://doi.org/10.1145/3131892

Duchowski, A. (2007). Eye Tracking Methodology. Londres: Springer London.

García Ogueta, M. (1991). Atención y sus cambios en el espacio visual. Cognitiva, 3(2), 205-236

Ens, B., Byagowi, A., Han, T., Hincapié-Ramos, J. D. \& Irani, P. (2016). Combining Ring Input with Hand Tracking for Precise, Natural Interaction with Spatial Analytic Interfaces. En Proceedings of the 2016 Symposium on Spatial User Interaction. (pp. 99-102). Nueva York: Association for Computing Machinery. DOI: 10.1145/2983310.2985757.

Ens, B., Hincapié-Ramos, J. D. \& Irani, P. (2014). Ethereal planes. En Proceedings of the 2nd ACM symposium on Spatial user interaction. (pp. 2-12). Nueva York: Association for Computing Machinery. DOI: 10.1145/2659766.2659769.

Eyraud, R., Zibetti, E. \& Baccino, T. (2015). Allocation of visual attention while driving with simulated augmented reality. Transportation Research Part F: Traffic Psychology and Behaviour, 32, 46-55.
DOI: 10.1016/j.trf.2015.04.011

Fast-Berglund, Å., Gong, L. \& Li, D. (2018). Testing and validating Extended Reality $(x \mathrm{R})$ technologies in manufacturing. Procedia Manufacture, 25, 31-38. DOI: 10.1016/j.promfg.2018.06.054.

Folker S. \&. Ritter, H. (2005). Proceedings of the Annual Meeting of the Cognitive Science Processing and Integrating Multimodal Material - The Influence of Color-Coding. Proceedings of the Annual Meeting of the Cognitive Science Society, 27. Recuperado de: https://escholarship.org/uc/item/5ch098t2

García-Sanjuan, F., Jaen, J. \& Nacher, V. (2016). Toward a General Conceptualization of Multi-Display Environments. Frontiers in ICT, 3. DOI: 10.3389/fict.2016.00020.

Harrison, R., Flood, D. \& Duce, D. (2013). Usability of mobile applications: literature review and rationale for a new usability model. Journal of interaction science, 1(1). DOI: 10.1186/21940827-1-1.

Hein, P., Bernhagen, M. \& Bullinger, A. C. (2019). Improving visual attention guiding by differentiation between fine and coarse navigation. 2019 11th International Conference on Virtual Worlds and Games for Serious Applications (VS-Games), Vienna. DOI: 10.1109/NS-Games.2019.8864539.

Heras, L. y Benitez, L. V. (2007). La realidad aumentada: una tecnología en espera de usuarios. Revista Digital Universitaria, 5(7).

Jang, W., Shin, J. H., Kim, M. \& Kim, K. (2016). Human field of regard, field of view, and attention bias. Computation methods programs biomedical, 135. DOI: 10.1016/j.cmpb.2016.07.026.

Johnson-Glenberg, M. C., Megowan-Romanowicz, C., Birchfield, D. A. \& Savio-Ramos, C. (2016). Effects of Embodied Learning and Digital Platform on the Retention of Physics Content: Centripetal Force. Frontiers in psychology, 7. DOI: 10.3389/ fpsyg.2016.01819.

Keebler, J. R. , Wiltshire, T. J., Smith, D. C., Fiore, S. M. \& Bedwell, J. S. (2014). Shifting the paradigm of music instruction: implications of embodiment stemming from an augmented reality guitar learning system. Frontiers in psychology, 5. DOI: 10.3389/ fpsyg.2014.00471.

Kishishita, N., Kiyokawa, K., Orlosky, J., Mashita, T., Takemura, H. \& Kruijff, E. (2014). Analysing the effects of a wide field of view 
augmented reality display on search performance in divided attention tasks. ISMAR 2014 IEEE International Symposium on Mixed and Augmented Reality (ISMAR), Munich. DOI: 10.1109/ ISMAR.2014.6948425.

Klimesch, W. (1999). EEG alpha and theta oscillations reflect cognitive and memory performance: a review and analysis. Brain Research Reviews, 29(2-3), 169-195. DOI: 10.1016/S0165-0173(98)00056-3.

de Koning, B., Tabbers, H. \& Paas, F. (2007). Attention Cueing as a Means to Enhance Learning from an Animation. Applied cognitive psychology, 22, 877-895. DOI: 10.1002/acp.1346.

Kruijff, E., Orlosky, J., Kishishita, N., Trepkowski, C. \& Kiyokawa, K. (2019). The Influence of Label Design on Search Performance and Noticeability in Wide Field of View Augmented Reality Displays. IEEE Transactions on Visualization and Computer Graphics, 25(9), 2821-2837. DOI: 10.1109/TVCG.2018.2854737.

Küçük, S., Kapakin, S. \& Göktaş, Y. (2016). Learning anatomy via mobile augmented reality: Effects on achievement and cognitive load, Anatomical science education, 9(5), 411-421. DOI: 10.1002/ ase.1603.

Ladouce, S., Donaldson, D. I., Dudchenko, P. A. \& letswaart, M. (2017). Understanding Minds in Real-World Environments: Toward a Mobile Cognition Approach. Frontiers in human neuroscience, 10. DOI: 10.3389/fnhum.2016.00694.

Lai, A. Chen, C. \& Lee, G. (2019). An augmented reality-based learning approach to enhancing students' science reading performances from the perspective of the cognitive load theory. British Journal of Education Technology, 50(1), 232-247. DOI: 10.1111/ bjet.12716.

Lou, C. I., Migotina, D., Rodrigues, J. P., Semedo, J., Wan, F., Mak, P. U., Mak, P. I., Mang, I. V., Melicio, F., Gomes, J. \& Rosa, A. (2012). Object recognition test in peripheral vision: A study on the influence of object color, pattern and shape. En F. M. Zanzotto, S. Tsumoto, N. Taatgen \& Y. Yao (eds). Brain Informatics. Lecture Notes in Computer Science. Vol. 7670. Berlín: Springer. https://doi. org/10.1007/978-3-642-35139-6_3

Lozano, E. M., Grau, P., Hernández, R., Benaloy, M. \& Ávila, C. (1995). Influencia de la expectativa sobre la orientación de la atención. Primeres Jornades de Foment de la Investigació de la FCHS.
Fòrum de recerca 1. Recuperado de: http://repositori.uji.es/xmlui/ bitstream/handle/10234/80627/forum_1995_13.pdf?sequence=1 u, W., Duh, B. L. \& Feiner, S. (2012). Subtle cueing for visual search in augmented reality. Proceedings of the 2012 IEEE International Symposium on Mixed and Augmented Reality. IIEEE Xplore, Atlanta. DOI:10.1109/ISMAR.2012.6402553.

Lu, W., Duh, H. B. L., Feiner, S. \& Zhao, Q. (2014). Attributes of subtle cues for facilitating visual search in augmented reality. IEEE Transactions on Visualization and Computer Graphics, 20(3), 404 412. DOI: 10.1109/TVCG.2013.241.

Lucas, N. \& Vuilleumier, P. (2008). Effects of emotional and nonemotional cues on visual search in neglect patients: Evidence for distinct sources of attentional guidance. Neuropsychologia, 46(5), 1401-1414. DOI: 10.1016/j. neuropsychologia.2007.12.027

Marek, N. \& Pollmann, S. (2020). Contextual-cueing beyond the initia field of view-a virtual reality experiment. Brain Science, 10(7) 1-9. DOI: 10.3390/brainsci10070446.

De Massari, D., Pacheco, D., Malekshahi, R., Betella, A., Verschure, P., Birbaumer, N. \& Caria, A. (2014). Fast mental states decoding in mixed reality. Frontiers of behavioral neurosciences, 8, 1-9. DOI: 10.3389/fnbeh.2014.00415.

Mcgill, M., Kehoe, A., Freeman, E. \& Brewster, S. (2020). Expanding the Bounds of Seated Virtual Workspaces. ACM Transactions on compututer-human interaction, 27(3), 1-40. DOI: 10.1145/3380959

Meyer, B. J. F. (1975). Organization of Prose and Its Effects on Memory. Amsterdam: North Holland Publications.

Moon, J. \& Ryu, J. (2020). The effects of social and cognitive cues on learning comprehension, eye-gaze pattern, and cognitive load in video instruction. Journal of computation in higher education 33, 39-63. DOI: 10.1007/s12528-020-09255-x.

Obradó Santaoliva, E. (2019). Tecnologías de realidad extendida aplicadas a la creación y a la docencia artística universitaria [Universidad de Sevilla]. In Universidad de Sevilla, Sevilla (Vol. 53, Issue 9). Tesis Doctoral Inédita

Obrado, Y. \& Spinola-Elias, E. (2019). Tecnologías de realidad extendida aplicadas a la creación y a la docencia artística 
universitaria. Journal of chemical information and modeling, 53(9), 1689-1699.

Ortega, M. (2013). 3D object position using automatic viewpoint transitions. En Proceedings of the SIGCHI Conference on Human Factors in Computing Systems. (pp. 193-196). Nueva York: Association for Computing Machinery. DOI: 10.1145/2470654.2470681.

Paas, F., Tuovinen, J. E., Tabbers, H. \& Van Gerven, P. (2003). Cognitive Load Measurement as a Means to Advance Cognitive Load Theory. Educational psychologist, 38(1), 63-71. DOI: 10.1207/ S15326985EP3801_8.

Posner, M. I. (2012). Attention in a Social World. Nueva York: Oxford University Press.

van Merriënboer, J. J. G. \& Sweller, J. (2005). Cognitive Load Theory and Complex Learning: Recent Developments and Future Directions. Education and psychology review, 17(2), 147-177. DOI: 10.1007/s10648-005-3951-0.

Rashid, U., Nacenta, M. A. \& Quigley, A. (2012a). Factors influencing visual attention switch in multi-display user interfaces. En Proceedings of the 2012 International Symposium on Pervasive Displays - PerDis '12, (pp.1-6). Nueva York: Association for Computing Machinery. DOI: 10.1145/2307798.2307799.

Rashid, U., Nacenta, M. A. \& Quigley, A. (2012b). The cost of display switching. En Proceedings of the International Working Conference on Advanced Visual Interfaces - AVI '12. Nueva York: Association for Computing Machinery. DOI: $10.1145 / 2254556.2254577$.

Renner, P. \& Pfeiffer, T. (2017a). Evaluation of attention guiding techniques for augmented reality-based assistance in picking and assembly tasks. Int. Conf. Intell. User Interfaces, Proc. IUI, pp. 89-92. DOI: 10.1145/3030024.3040987.

Renner, P. \& Pfeiffer, T. (2017b). Attention guiding techniques using peripheral vision and eye tracking for feedback in augmented-reality-based assistance systems. IEEE Symposium on 3D User Interfaces (3DUI), pp. 186-194. DOI: 10.1109/3DUI.2017.7893338.

Rojas-Murillo, S. \& Pennathur, P. R. (2019). Selection of key visual cues in real and virtual environments for assembly tasks. International
Journal of Industrial Ergonomics, 74. DOI: 10.1016/j. ergon.2019.102871.

Schroeder, N. L. \& Cenkci, A. T. (2018). Spatial Contiguity and Spatial Split-Attention Effects in Multimedia Learning Environments: a Meta-Analysis. Educational psychology review, 30(3), 679-701. DOI: 10.1007/s10648-018-9435-9.

Soret, R., Charras, P., Hurter, C. \& Peysakhovich, V. (2019). Attentional orienting in virtual reality using endogenous and exogenous cues in auditory and visual modalities. Nueva York: Association for Computing Machinery. DOI: 10.1145/3314111.3321490.

Smith, S. P. \& Hart, J. (2006). Evaluating distributed cognitive resources for wayfinding in a desktop virtual environment. 3D User Interfaces. Alexandria. DOI: 10.1109/VR.2006.60.

Sweller, J., van Merrienboer, J. \& Paas, F. (1998). Cognitive Architecture and Instructional Design. Educational psychology review, 10, 251-296. DOI: https://doi.org/10.1023/A:1022193728205.

Taylor, H. A., Rapp, D. N. \& Brunye, T. A. (2007). Repetition and Dual Coding in Procedural Multimedia Presentations. Applied cognitive psychology, 22, 877-895. DOI: 10.1002/acp.

Taylor, P., Bilgrien, N., He, Z. \& Siegelmann, H. T. (2015). EyeFrame: Real-time memory aid improves human multitasking via domain-general eye tracking procedures. Frontiers. ICT, 2, 1-13. DOI: 10.3389/fict.2015.00017.

Turan, Z., Meral, E. \& Sahin, I. F. (2018). The impact of mobile augmented reality in geography education: Achievements, cognitive loads and views of university students. Journal in geography in higher education, 42(3) 427-441. DOI: 10.1080/03098265.2018.1455174.

Van Dam, J., Krasner, A. \& Gabbard, J. L. (2020). Drone-based Augmented Reality Platform for Bridge Inspection: Effect of AR Cue Design on Visual Search Tasks. Proc. - 2020 IEEE Conf. Virtual Real. 3D User Interfaces, VRW 2020, DOI: 10.1109/ VRW50115.2020.00043.

Volmer, B, Baumeister, J., Von Itzstein, S., Bornkessel-Schlesewsky, I. \& Schlesewsky, M. (2018). A comparison of predictive spatial augmented reality cues for procedural tasks. IEEE Transactions on Visualization and Computer Graphics, 24(11), 2846-2856. DOI: 10.1109/TVCG.2018.2868587. 
Wang, X., Ong, S. K. \& Nee, A. Y. C. (2016). Multi-modal augmentedreality assembly guidance based on bare-hand interface. Advance engineering informatics, 30(3), 406-421. DOI: 10.1016/j.aei.2016.05.004.

Zhao, X. ,Shi, C., You, X. \& Zong, C. (2017). Analysis of Mental Workload in Online Shopping: Are Augmented and Virtual Reality Consistent? Frontiers in psychology, 8. DOI: 10.3389/ fpsyg.2017.00071. 